\title{
VDTA Based Electronically Tunable Voltage-Mode and Trans-Admittance Biquad Filter
}

\author{
Gunjan Gupta1, Sajai Vir Singh ${ }^{2}$, Sunil Vidya Bhooshan ${ }^{1}$ \\ ${ }^{1}$ Department of Electronics and Communication Engineering, Jaypee University of Information Technology, \\ Solan, India \\ ${ }^{2}$ Department of Electronics and Communication Engineering, Jaypee Institute of Information Technology, \\ Noida, India \\ Email: gunjan.techno@gmail.com, sajaivir@rediffmail.com, sunil.bhooshan@juit.ac.in
}

Received 18 November 2014; accepted 26 March 2015; published 27 March 2015

Copyright (C 2015 by authors and Scientific Research Publishing Inc.

This work is licensed under the Creative Commons Attribution International License (CC BY).

http://creativecommons.org/licenses/by/4.0/

(c) $\underset{\mathrm{EY}}{\mathrm{Er}}$ 0pen Access

\begin{abstract}
In this paper, a biquad filter configuration based on two voltage differencing transconductance amplifiers (VDTAs) as newly active elements and only two capacitors as passive elements is proposed which can realize voltage-mode low pass (LP), band pass (BP), high pass (HP), band reject (BR) and all pass (AP) filtering responses using three voltage inputs. Simultaneously, the same configuration can also be used to obtain LP, BP and HP filtering responses in transadmittancemode. The proposed biquad is capable of providing electronic control of quality factor independent of pole frequency through single transconductance parameter (biasing current). It also offers the advantage of low active and passive sensitivity. To support the theoretical analysis, the PSPICE simulation of the proposed circuit is done using $0.18 \mu \mathrm{m}$ CMOS technology from TSMC.
\end{abstract}

\section{Keywords}

VDTA, Biquad Filter, Voltage-Mode, Trans-Admittance-Mode

\section{Introduction}

In the last few decades, current-mode active elements have been preferred over voltage-mode active elements in the designing of high performance continuous time analog filters [1] due to their several salient features such as inherently wider bandwidth, greater linearity, wider dynamic range, simple circuitry and low power consumptions. Consequently, several current-mode active elements such as second generation current conveyor (CCII), 
differential difference current conveyor (DDCCII), current differencing buffer amplifier (CDBA), operational transconductance amplifier (OTA), current controlled current conveyor (CCCII), current differencing transconductance amplifier (CDTA), current controlled current differencing transconductance amplifier (CCCDTA), current controlled current conveyor transconductance amplifier (CCCCTA), voltage differencing transconductance amplifier (VDTA) etc. and their applications in filters design are introduced in the literature [2]-[26]. Among them VDTA is latest one. It has the advantage of its electronic tuning ability through two transconductance parameters; hence it does not need a resistor in practical applications. Subsequently, the VDTA based circuits realizations occupy less chip area. This device can be operated in both current- and voltage-modes, providing flexibility to the circuit designers [25].

Literature survey shows that quite a number of VDTA based biquad filter either as single-input multi-output (SIMO) and/or as multi-input single-output (MISO) types have been reported in the available literature till date [19]-[26]. But as far as the topic of this paper is concerned, the filter circuits operated in either voltage-mode or trans-admittance-mode-mode or in both modes simultaneously, using VDTAs, are of interest. The reported voltagemode filter based on VDTA [22]-[24] uses only single VDTA and three (two capacitors and one resistors) [22] or two passive elements (only two capacitors) [23] [24]. Two of the circuits [22] [23] can realize all the standard filtering functions (LP, BP, HP, BR and AP) by the use of three inputs and single-output. However, both circuits are not able to provide orthogonal electronic tunability of pole frequency and quality factor. Moreover, the circuit [22] requires inverting type voltage input to realize AP filtering function. Third of the circuit [24] can realizes only three filtering functions (LP, BP and HP) by the use of two inputs and two outputs. The single-input five-output biquad filter reported in Ref. [25] employs two VDTAs, two grounded capacitors, two grounded resistors and realizes all the five standard filtering functions, simultaneously, in voltage-mode only with feature of orthogonal electronic tunabilty of pole frequency and quality factor. Another valuable filter circuit with single-input multi-output [26] consists of two VDTAs, two capacitors and realizes only two filtering functions (LP, BP) in voltage-mode and three filtering functions (BP, LP and BR) in trans-admittance-mode, simultaneously.

Considering the above facts, a new biquad filter configuration is proposed in this paper. The proposed configuration can realize BP, HP, LP, RN, and AP responses in voltage-mode as three-input single-output structure and LP, BP, HP responses in trans-admittance-mode as single-input three-output structure. The configuration comprises of only two VDTAs, two capacitors as active and passive elements, respectively and does not require 1) external resistor(s), and 2) minus and/or double type voltage input signal(s) to realize any filtering response. Moreover, it has less active and passive sensitivity.

\section{Description of VDTA and Proposed Filter Configuration}

Voltage differencing transconductance amplifiers is relatively new active element [24] [25] whose symbolic diagram is shown in Figure 1 where $P, N$ are two high impedance input terminals and $Z, Z c, X^{+}, X^{-}$are the high impedance output terminals. The differential voltage across high impedance input terminals $P$ and $N\left(V_{P}-V_{N}\right)$ is transferred to a current at high impedance output terminals $Z$ and $Z c\left(I_{Z}\right.$ and $\left.I_{Z c}\right)$ by transconductance parameters $\left(g_{m}^{F}\right)$ of VDTA. Further voltage across auxiliary $Z$ terminal is also transferred to a current at terminals $X^{+}$and

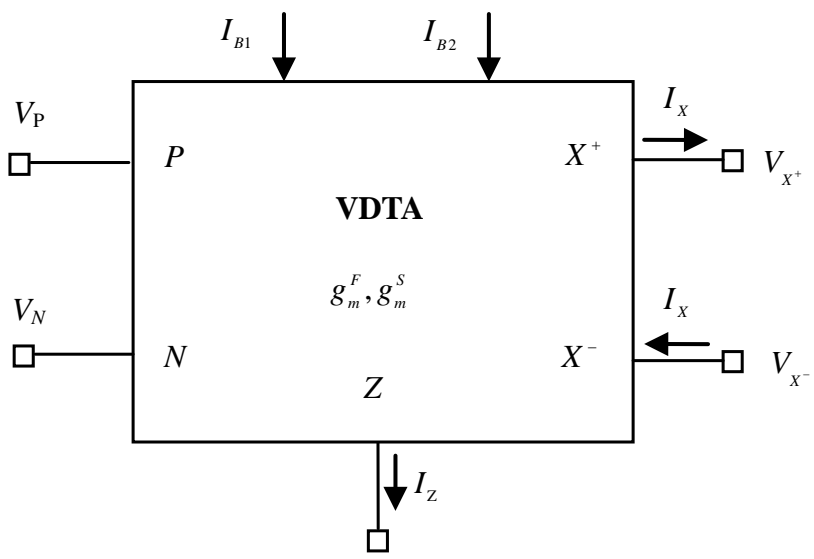

Figure 1. Symbolic diagram of VDTA. 
$X^{-}\left(I_{X^{+}}\right.$and $\left.I_{X^{-}}\right)$by other transconductance parameters $\left(g_{m}^{S}\right)$ of VDTA. The $g_{m}^{F}$ and $g_{m}^{S}$ are the transconductance parameters of first and second stage of VDTA respectively, whose value are controlled by biasing currents $I_{B}^{F}$ and $I_{B}^{S}$ of VDTA, respectively. The relationship of input output terminals of VDTA can be described by the following matrix equation.

$$
\left[\begin{array}{c}
I_{Z}, I_{Z c} \\
I_{X^{+}} \\
I_{X^{-}}
\end{array}\right]=\left[\begin{array}{ccc}
g_{m}^{F} & -g_{m}^{S} & 0 \\
0 & 0 & g_{m}^{S} \\
0 & 0 & -g_{m}^{S}
\end{array}\right]\left[\begin{array}{c}
V_{P} \\
V_{N} \\
V_{Z}
\end{array}\right]
$$

The CMOS realization of VDTA is also shown in Figure 2 which consists of two Arbel-Goldminz transconductance [25]. For the CMOS implementation of VDTA as shown in Figure 2, $g_{m}^{F}$ and $g_{m}^{S}$ can be approximately expressed by the following equations [25].

$$
g_{m}^{F} \cong\left(\frac{g_{1} g_{2}}{g_{1}+g_{2}}\right)+\left(\frac{g_{3} g_{4}}{g_{3}+g_{4}}\right)
$$

and

$$
g_{m}^{S} \cong\left(\frac{g_{5} g_{6}}{g_{5}+g_{6}}\right)+\left(\frac{g_{7} g_{8}}{g_{7}+g_{8}}\right)
$$

where $g_{i}=\sqrt{I_{B i} \mu C_{O X} \frac{W_{i}}{L_{i}}}$ is transconductance gain of $i^{\text {th }}$ transistor $(i=1,2,3,4,5,6,7,8) . I_{B i}, W_{i}, L_{i}$ are the bias current, effective channel width and length of $i^{\text {th }}$ MOS transistor, respectively. $\mu$ is the effective carrier mobility and $C_{O X}$ is the gate oxide capacitance per unit area of the MOS transistors.

The proposed biquad filter configuration is shown in Figure 3, which employs two VDTAs and two capacitors with one is permanently grounded. The analysis of the circuit of Figure 3 for three applied voltage inputs $V_{1}$, $V_{2}$ and $V_{3}$, will give the following expression for the voltage output at $V_{0}$

$$
V_{0}=\frac{V_{3} s^{2} C_{1} C_{2}-V_{2} s C_{1} g_{m 2}^{F}+V_{1} g_{m 1}^{F} g_{m 2}^{F}}{D(s)}
$$

where

$$
D(s)=s^{2} C_{1} C_{2}+s C_{1} g_{m 2}^{S}+g_{m 1}^{F} g_{m 2}^{F}
$$

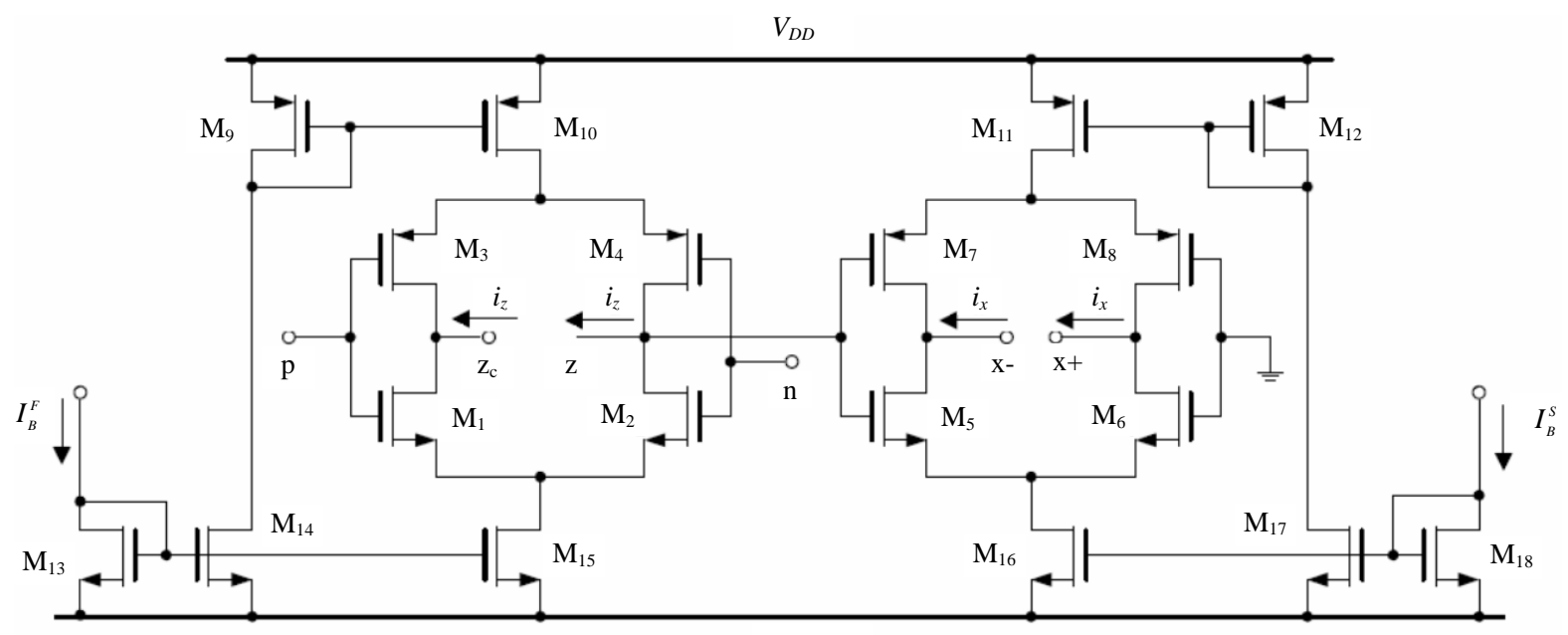

$-V_{S S}$

Figure 2. CMOS implementation of VDTA. 


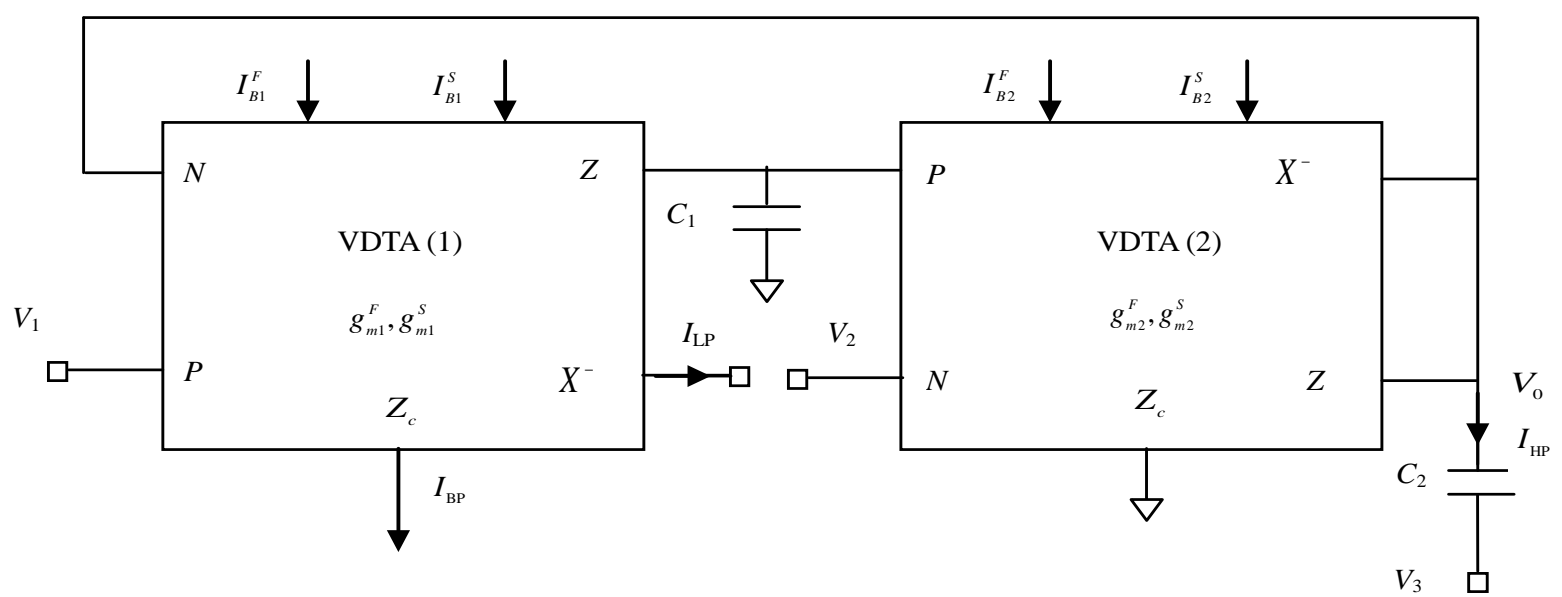

Figure 3. The proposed biquad filter configuration based on VDTA.

Here $g_{m 1}^{F}, g_{m 1}^{S}$ are transconductance gains of first and second stage of VDTA (1) respectively and $g_{m 2}^{S}$, $g_{m 2}^{s}$ are transconductance gains of first and second stage of VDTA (2), respectively. It is evident from Equation (4) that various biquad filtering responses in voltage-mode can be obtained at $V_{0}$ through appropriate selection of input voltage signals $\left(V_{1}, V_{2}\right.$, and $\left.V_{3}\right)$.

1) If $V_{1}=V_{\text {in }}$ and $V_{2}=V_{3}=0$, the filter configuration provides LP response.

2) If $V_{2}=V_{\text {in }}$ and $V_{1}=V_{3}=0$, the filter configuration provides BP response.

3) If $V_{3}=V_{\text {in }}$ and $V_{1}=V_{2}=0$, the filter configuration provides HP response.

4) If $V_{1}=V_{3}=V_{\text {in }}, V_{2}=0$, the filter configuration provides BR response.

5) If $V_{\text {in } 1}=V_{\text {in2 }}=V_{\text {in } 3}=V_{\text {in }}$ and $g_{m 2}^{S}=g_{m 2}^{F}$, the filter configuration provides AP responses.

It can be concluded from above operational description that the proposed filter configuration in Figure 3 is capable of realizing all the five standard filtering responses in voltage-mode without requiring minus-type and/or double input voltage signal(s). However, AP realization requires matching condition.

In addition to above voltage mode filtering responses, the same configuration can also be used to realize LP, $\mathrm{BP}$, HP transadmittance mode responses across $I_{\mathrm{LP}}, I_{\mathrm{BP}}$ and $I_{\mathrm{HP}}$, respectively, by applying only one voltage input $V_{2}\left(V_{\text {in }}\right)$ and remaining voltage inputs set to zero $\left(V_{1}=V_{3}=0\right)$. In this case transadmittance mode transfer function can be derived as

$$
\begin{gathered}
\frac{I_{\mathrm{BP}}}{V_{\mathrm{in}}}=\frac{s C_{1} g_{m 1}^{F} g_{m 2}^{F}}{D(s)} \\
\frac{I_{\mathrm{LP}}}{V_{\mathrm{in}}}=\frac{g_{m 1}^{F} g_{m 1}^{S} g_{m 2}^{F}}{D(s)} \\
\frac{I_{\mathrm{HP}}}{V_{\mathrm{in}}}=\frac{s^{2} C_{1} C_{2} g_{m 2}^{F}}{D(s)} .
\end{gathered}
$$

The filter characteristic parameters like pole-frequency $\left(\omega_{0}\right)$, quality-factor $\left(Q_{0}\right)$, and band-width $\left(\omega_{0} / Q_{0}\right)$ for the proposed filter configuration can be expressed

$$
\begin{aligned}
& \omega_{0}=\sqrt{\frac{g_{m 1}^{F} g_{m 2}^{F}}{C_{1} C_{2}}} \\
& Q_{0}=\frac{1}{g_{m 2}^{S}} \sqrt{\frac{g_{m 1}^{F} g_{m 2}^{F} C_{2}}{C_{1}}} \\
& \mathrm{BW}=\frac{\omega_{0}}{Q_{0}}=\frac{g_{m 2}^{S}}{C_{2}} .
\end{aligned}
$$


If $g_{m 1}^{F}=g_{m 2}^{F}=g_{m}^{F}$ and $C_{1}=C_{2}=C$, then Equation (11) becomes

$$
\omega_{0}=\frac{g_{m}^{F}}{C}, \quad \mathrm{BW}=\frac{\omega_{0}}{Q_{0}}=\frac{g_{m 2}^{S}}{C}
$$

and

$$
Q_{0}=\frac{g_{m}^{F}}{g_{m 2}^{s}}
$$

It is clear from Equation (10) and Equation (11) that filter parameter $Q_{0}$ can be widely varied electronically by varying $g_{m 2}^{S}$ independent of $\omega_{0}$. Similarly, $\omega_{0}$ and BW are electronically orthogonal tunable.

\section{Non Ideal and Sensitivity Analysis}

For non ideal characteristics of the VDTA, the port relations of currents and voltages in Equation (1) can be changed as follow.

$$
\left[\begin{array}{c}
I_{Z}, I_{Z c} \\
I_{X^{+}} \\
I_{X^{-}}
\end{array}\right]=\left[\begin{array}{ccc}
\beta_{j}^{F} g_{m j}^{F} & -\beta_{j}^{F} g_{m j}^{F} & 0 \\
0 & 0 & \beta_{j}^{F} g_{m j}^{F} \\
0 & 0 & -\beta_{j}^{F} g_{m j}^{F}
\end{array}\right]\left[\begin{array}{c}
V_{P} \\
V_{N} \\
V_{Z}
\end{array}\right]
$$

where $\beta_{j}^{F}$ and $\beta_{j}^{S}$ represents the voltage tracking errors for first and second stages of $j^{\text {th }}$ VDTA respectively, where $j=1$, 2. If we re-analysed the proposed circuit in Figure 3 using Equation (13), the filter parameters $\omega_{0}$, $Q_{0}$, and BW are changed to

$$
\omega_{0}=\sqrt{\frac{\beta_{1}^{F} g_{m 1}^{F} \beta_{2}^{F} g_{m 1}^{S}}{C_{1} C_{2}}}, \quad \mathrm{BW}=\frac{\omega_{0}}{Q_{0}}=\frac{\beta_{2}^{S} g_{m 2}^{S}}{C_{2}}, \text { and } Q_{0}=\frac{1}{\beta_{2}^{S} g_{m 2}^{S}} \sqrt{\frac{\beta_{1}^{F} g_{m 1}^{F} \beta_{2}^{F} g_{m 1}^{S} C_{2}}{C_{1}}}
$$

The active and passive relative sensitivities of $\omega_{0}$ and $Q_{0}$ for the proposed filter are calculated as

$$
\begin{aligned}
& S_{g_{m 1}^{F}}^{\omega_{0}}=S_{g_{m 1}^{S}}^{\omega_{0}}=S_{\beta_{1}^{F}}^{\omega_{0}}=S_{\beta_{2}^{F}}^{\omega_{0}}=\frac{1}{2}, S_{C_{2}}^{\omega_{0}}=S_{C_{1}}^{\omega_{0}}=-\frac{1}{2}, S_{g_{m 2}^{F}, g_{m 2}^{S}}^{\omega_{0}}=S_{\beta_{1}^{S}, \beta_{2}^{S}}^{\omega_{0}}=0 \\
& S_{g_{m 1}^{F}}^{Q_{0}}=S_{g_{m 1}^{S}}^{Q_{0}}=S_{\beta_{1}^{F}}^{Q_{0}}=S_{\beta_{2}^{F}}^{Q_{0}}=\frac{1}{2}, S_{C_{2}}^{Q_{0}}=-S_{C_{1}}^{\omega_{0}}=\frac{1}{2}, S_{g_{m 2}^{F}}^{Q_{0}}=S_{\beta_{1}^{S}, \beta_{2}^{S}}^{Q_{0}}=0
\end{aligned}
$$

and

$$
S_{g_{m 2}^{s}}^{Q_{0}}=S_{\beta_{2}^{s}}^{Q_{0}}=-1 .
$$

From Equation (15)-(17), it is clear that the proposed circuit possess low active and passive sensitivities, less than or equal to unity in magnitude.

\section{Simulation Results}

In this section the performance of the proposed biquad filter configuration of Figure 3 was examined using PSPICE simulations. Simulation was performed based on CMOS structure of VDTA as shown in Figure 2, with transistor model of $0.18 \mu \mathrm{m}$ MOSFET from TSMC [27]. Aspect ratio of each MOS transistors is given in Table 1. To design the proposed biquad filter for obtaining $f_{o}=\omega_{0} / 2 \pi=7.7 \mathrm{MHZ}$ and $Q_{0}=1$, the active and passive components were selected as $C_{1}=C_{2}=10 \mathrm{pF}, V_{D D}=-V_{S S}=2 \mathrm{~V}, I_{B 1}^{F}=I_{B 1}^{S}=I_{B 2}^{F}=I_{B 2}^{S}=50 \mu \mathrm{A}$, $\left(g_{m 1}^{F}=g_{m 2}^{F}=g_{m 1}^{S}=g_{m 2}^{S}=484 \mu \mathrm{A}\right)$. Figure 4 shows the voltage-mode gain and phase responses of LP, BP, HP, BR and AP. Figure 5 shows the transadmittance gain of LP, BP and HP filtering responses. The simulation result shows the simulated pole frequency as $7.49 \mathrm{MHz}$ which is fairly closed to the designed value of $7.7 \mathrm{MHz}$. The total power dissipation for the circuit is $2.71 \mathrm{~mW}$. Figure 6 shows the electronic tunability feature of $Q_{0}$ independent of $\omega_{0}$ for the proposed filter by performing the simulation of various voltage-mode BP responses at 


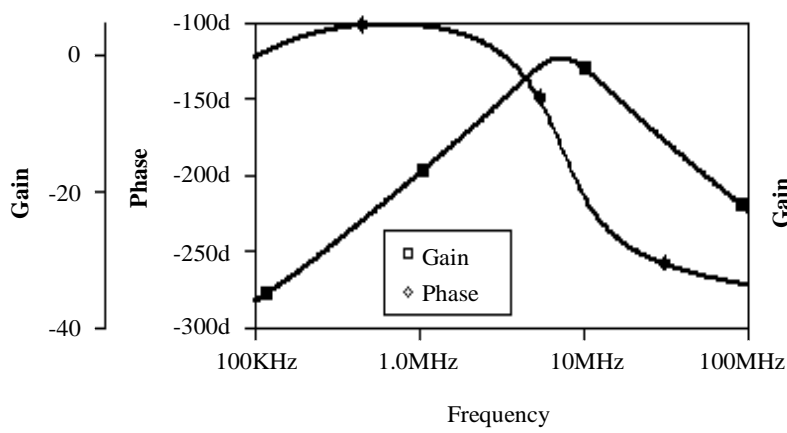

(a)

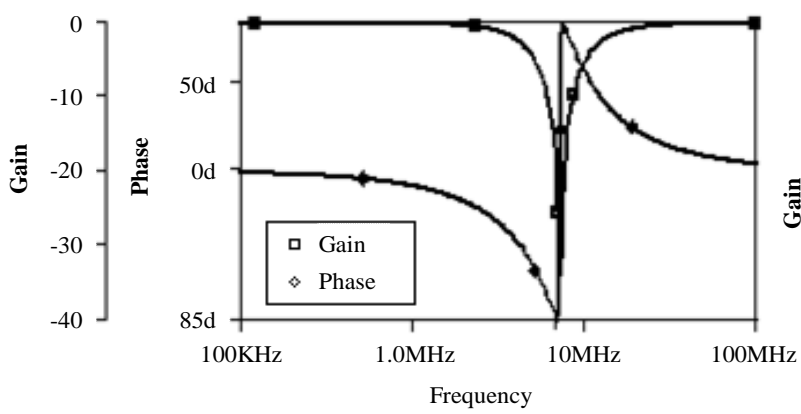

(c)
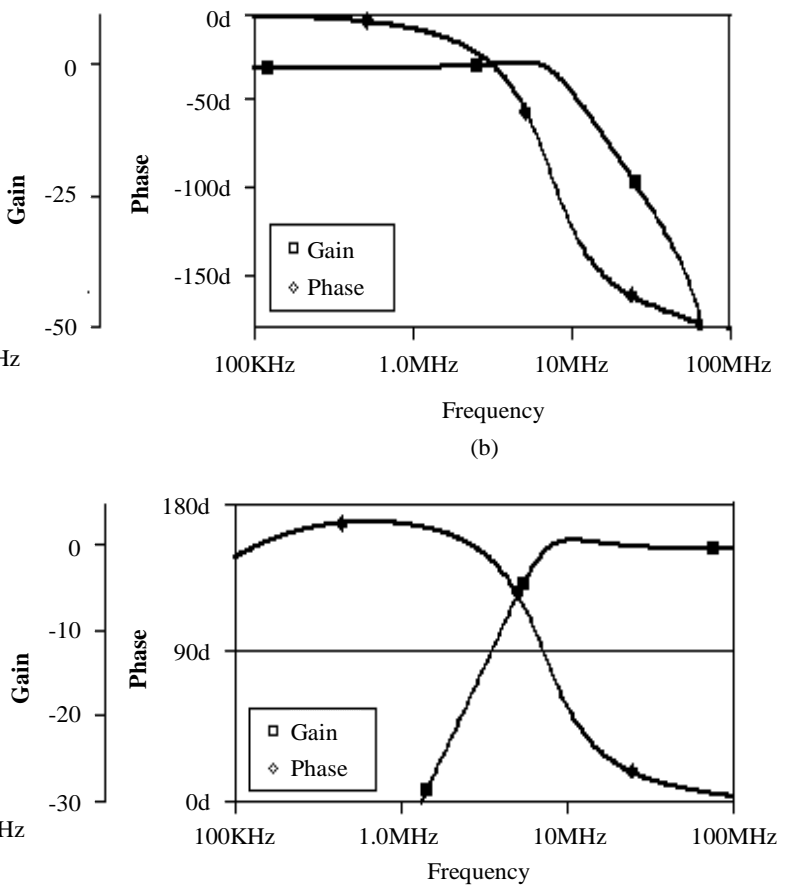

(d)

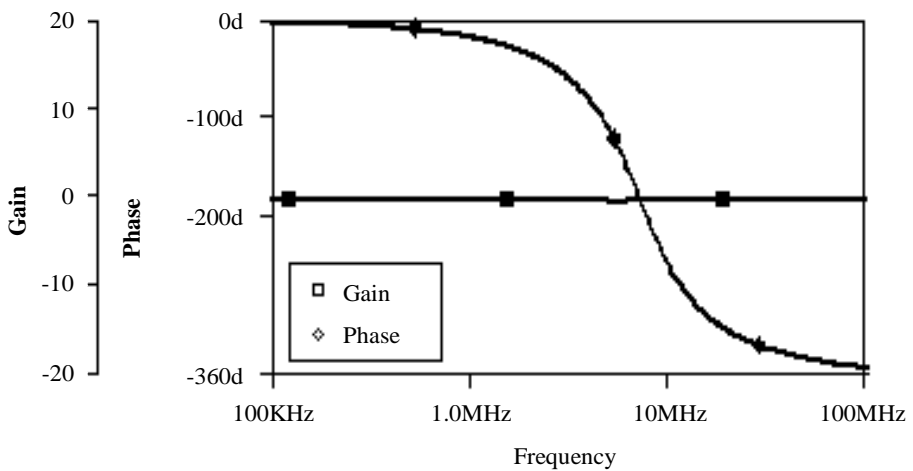

(e)

Figure 4. Simulated gain and phase response of the proposed voltage-mode filter: (a) Band-pass; (b) Low-pass; (c) Bandreject; (d) High-pass; and (e) All-pass.

different values of $I_{B 2}^{S}=5 \mu \mathrm{A}, 20 \mu \mathrm{A}, 60 \mu \mathrm{A}, 100 \mu \mathrm{A}$ by keeping other currents as constant of $50 \mu \mathrm{A}$. The corresponding quality factor at constant pole frequency of 7.49 were found as $Q_{0}=3.14,1.58,0.909,0.707$ which prove the electronic tunability feature of $Q_{0}$ independent of $\omega_{0}$ for the proposed filter. Now, the noise effect for the proposed filter is considered by showing the voltage-mode BP output noise spectral density in Figure 7. It indicates that noise spectral density is quite small and it attains a maximum value equal to $14 \mathrm{nV} / \mathrm{Hz}^{1 / 2}$. Further, Monte-Carlo analysis is also performed to perceive the effect of capacitive deviations on the performance of proposed circuit. The voltage-mode BP response has been simulated with $10 \%$ Gaussian deviation in $C_{1}=C_{2}$ $=10 \mathrm{pF}$. The simulation was done simultaneously for 100 runs. The corresponding result is shown in Figure 8 . From this result, the standard deviation is measured as $292.42 \mathrm{kHz}$, which demonstrates that the circuit is reasonable sensitive towards capacitive passive components. For 5\% Gaussian deviation, the sensitivity is 145.35 $\mathrm{kHz}$. Lastly, the time domain behaviour of the proposed filter in Figure 3 was also investigated by applying a sinusoidal input voltage having peak to peak amplitude of $300 \mathrm{mV}$ at a signal frequency of $200 \mathrm{KHz}$. Figure 9 shows the time domain sinusoidal voltage input $\left(V_{1}=V_{\text {in }}\right)$ and corresponding LP voltage output signal. From Figure 9, it was observed that $300 \mathrm{mV}$ peak to peak sinusoidal input voltage signal of frequency $200 \mathrm{KHz}$ is acceptable without significant distortions. 


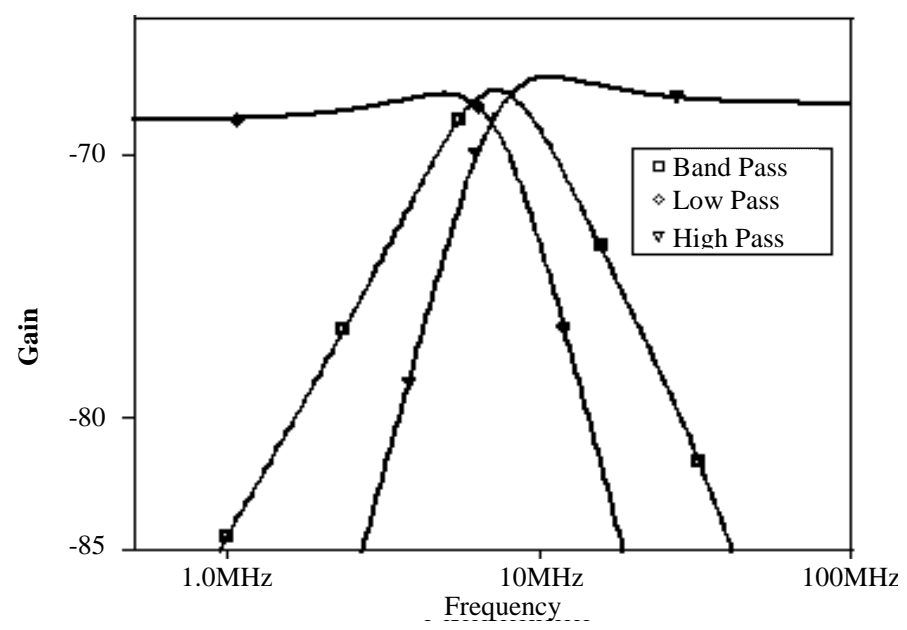

Figure 5. Transadmittance mode filter responses.

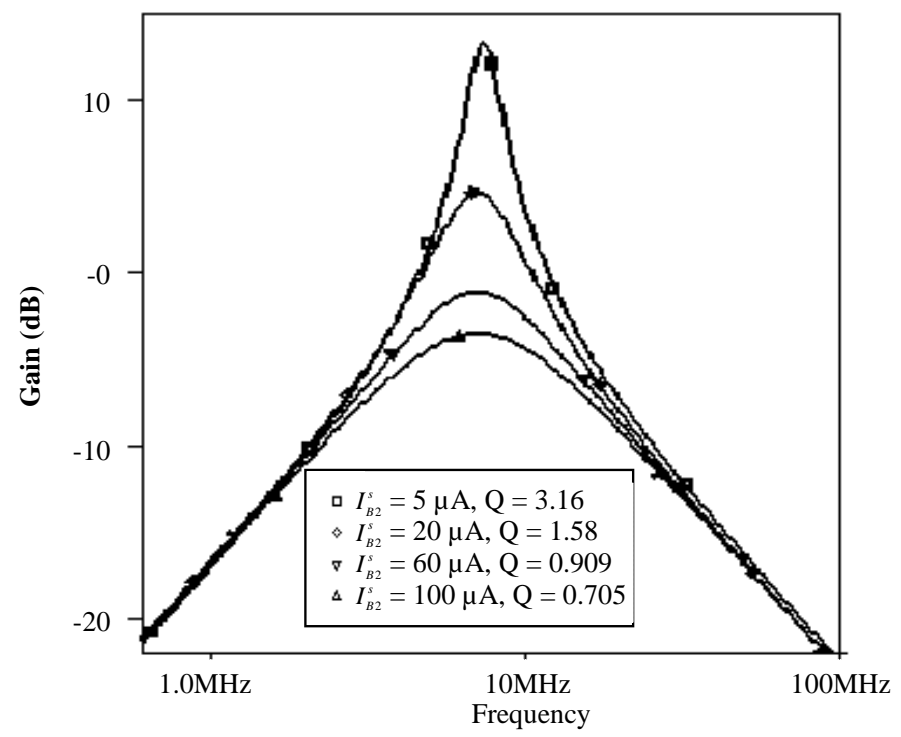

Figure 6. Band pass responses for different values of $I_{B 2}^{S}$.

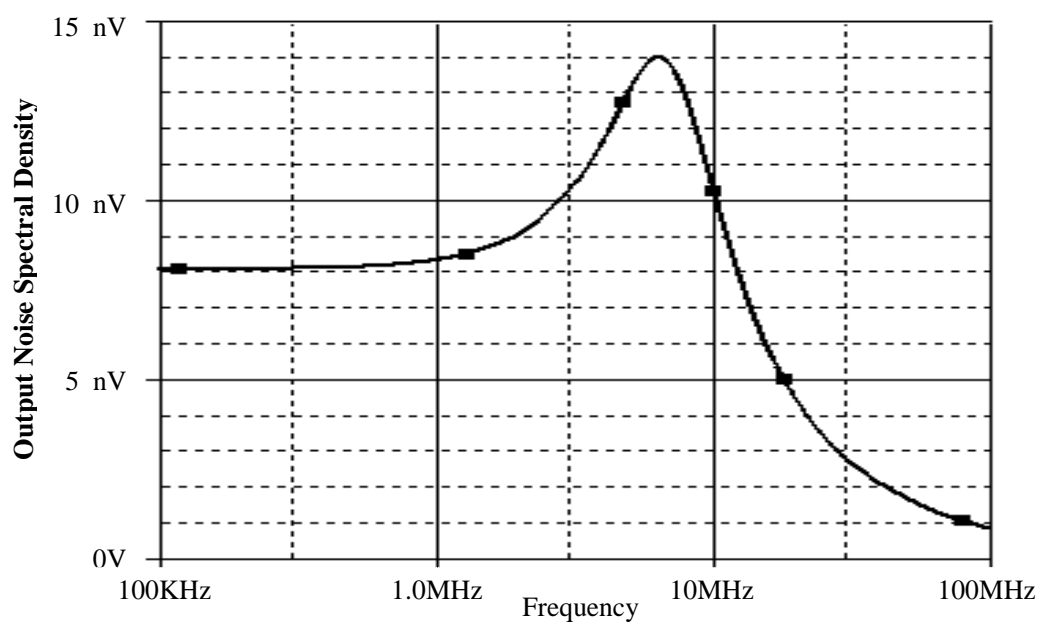

Figure 7. Output noise spectral density of the BP response of the proposed voltage mode circuit. 


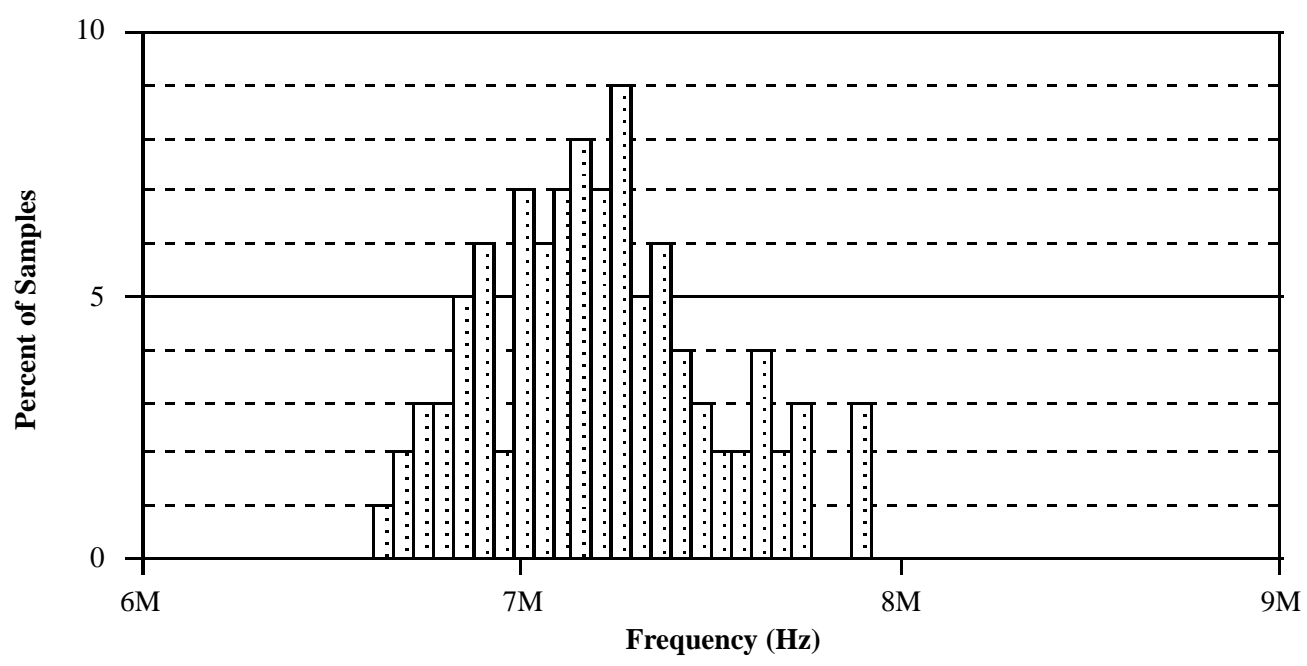

\begin{tabular}{|llll|}
\hline $\mathrm{n}$ samples & $=100$ & mean & $=7.20479 \mathrm{e}+006$ \\
$\mathrm{n}$ divisions & $=25$ & sigma & $=292424$ \\
median & $=7.18582 \mathrm{e}+006$ & minimum & $=6.60806 \mathrm{e}+006$ \\
90th \%ile & $=7.63673 \mathrm{e}+006$ & 10 th \%ile & $=6.82291 \mathrm{e}+006$ \\
maximum & $=7.92319 \mathrm{e}+006$ & $3 *$ sigma & $=877271$ \\
\hline
\end{tabular}

Figure 8. Monte-Carlo analysis for the voltage-mode BP response with $10 \%$ deviation in capacitor values.
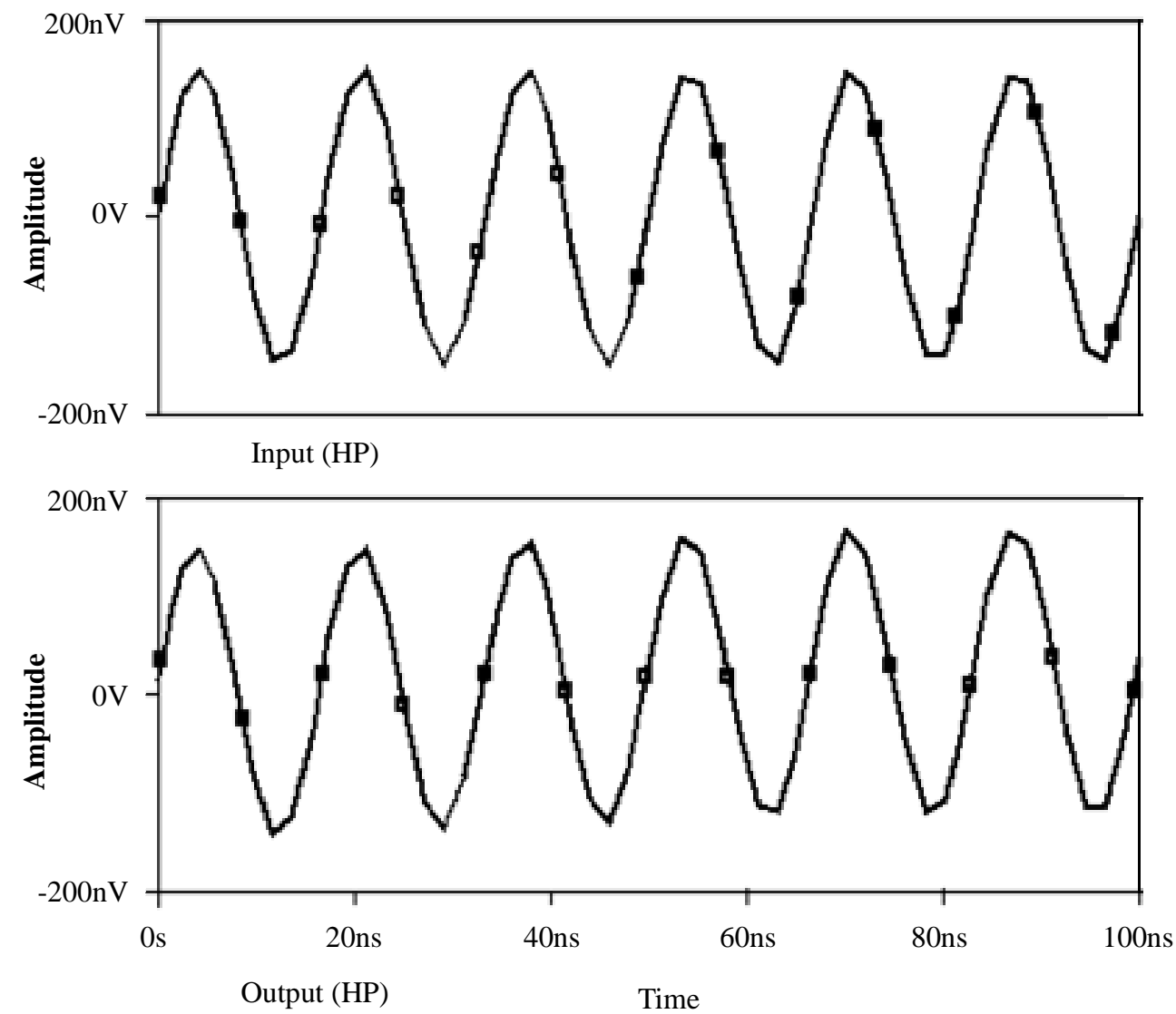

Figure 9. Time-domain results of LP response for transient analysis. 


\section{Conclusions}

This paper has presented a biquad filter configuration based on VDTAs which employs two VDTAs and two capacitors and offers the following attractive features:

1) Capable of realizing LP, BP, HP, BR, and AP filtering responses in voltage-mode and LP, BP, and HP filtering responses in trans-admittance-mode from the same configuration.

2) No employment of resistor(s), hence suited for integration.

3) The circuit is canonical by the way of using only two capacitors.

4) No need of minus type and/or double type input voltage signal to realize any filtering response, hence make the circuit simpler.

5) $Q_{0}$ control of independent of $\omega_{0}$ through single transconductance parameter (biasing current), hence suited for practical applications.

6) Low active and passive sensitivity performance.

7) Low power consumptions.

With above mentioned features and exhaustive simulation results, it is very suitable to implement the proposed filter circuit in monolithic chip for use in modern microelectronic system applications, such as controls, voice and data communications.

\section{References}

[1] Toumazou, C., Lidgey, F.J. and Marks, C.A. (1990) Extending Voltage-Mode Op-Amps to Current-Mode Performance. IEE Proceedings G: Circuits, Devices and Systems, 137, 116-129. http://dx.doi.org/10.1049/ip-g-2.1990.0020

[2] Sedra, A.S. and Smith, K.C. (1970) A Second Generation Current Conveyor and Its Application. IEEE Transactions on Circuit Theory, 17, 132-134. http://dx.doi.org/10.1109/TCT.1970.1083067

[3] Soliman, A.M. (2007) New Current-Mode Bandpass Filters Using Three Single Output ICCIIs. Active and Passive Electronic Components, Article ID: 69515.

[4] Singh, V.K. and Senani, R. (1990) New Multifunction Active Filter Configuration Employing Current Conveyors. Electronics Letters, 26, 1814-1816. http://dx.doi.org/10.1049/el:19901161

[5] Abuelma'atti, M.T. and Al-Zahar, H.A. (1997) New Universal Filter Using Two Current-Feedback Amplifiers. Active and Passive Electronic Components, 20, 111-117. http://dx.doi.org/10.1155/1997/39352

[6] Maheshwari, S. (2009) Analogue Signal Processing Applications Using a New Circuit Topology. IET Circuits, Devices and Systems, 3, 106-115. http://dx.doi.org/10.1049/iet-cds.2008.0294

[7] Horng, J.W., Chiu, W.Y. and Wei, H.Y. (2004) Voltage-Modes High Pass, Band Pass and Low Pass Filters Using Two DDCCIIs. International Journal of Electronics, 91, 461-464. http://dx.doi.org/10.1080/00207210412331294603

[8] Minaei, S. and Türköz, S. (2004) Current-Mode Electronically Tunable Universal Filter Using Only Plus-Type Current Controlled Conveyors and Grounded Capacitors. ETRI Journal, 26, 292-296. http://dx.doi.org/10.4218/etrij.04.0103.0146

[9] Chiu, W.Y. and Horng, J.W. (2007) High Input and Low Output Impedance Voltage Mode Universal Biquadratic Filter Using DDCCs. IEEE Transactions on Circuits and Systems II: Analog and Digital Signal Processing, 54, 649-652. http://dx.doi.org/10.1109/TCSII.2007.899460

[10] Abuelma'atti, M.T. (2003) A Novel Mixed-Mode Current-Controlled Current Conveyor-Based Filter. Active Passive Electronic Components, 26, 185-191. http://dx.doi.org/10.1080/1042015031000073841

[11] Bhaskar, D.R., Singh, A.K., Sharma, R.K. and Senani, R. (2005) New OTA-C Universal Current-Mode/Trans-Admittance Biquads. IEICE Electronic Express, 2, 8-13. http://dx.doi.org/10.1587/elex.2.8

[12] Lee, C.N. (2009) Multiple-Mode OTA-C Universal Biquad Filter. Circuits Systems and Signal Processing, 29, $263-274$. http://dx.doi.org/10.1007/s00034-009-9145-0

[13] Keskin, A.Ü., Biolek, D., Hancioglu, E. and Biolkova, V. (2006) Current-Mode KHN Filter Employing Current Differencing Transconductance Amplifiers. International Journal of Electronics and Communications (AEÜ), 60, 443-446.

[14] Biolek, D. and Biolkova, V. (2003) CDTA-C Current-Mode Universal 2nd Order Filter. 5th International Conference on Applied Informatics and Communications, 411-414.

[15] Siripruchyanun, M. and Jaikla, W. (2008) CMOS Current Controlled Current Differencing Transconductance Amplifier and Applications to Analog Signal Processing. International Journal of Electronics and Communications $(A E \ddot{)}), \mathbf{6 2}$, 277-287. http://dx.doi.org/10.1016/j.aeue.2007.05.001

[16] Singh, S.V., Maheshwari, S., Mohan, J. and Chauhan, D.S. (2009) An Electronically Tunable SIMO Biquad Filter Us- 
ing CCCCTA. Communications in Computer and Information Science, 40, 544-555. http://dx.doi.org/10.1007/978-3-642-03547-0_52

[17] Maheshwari, S., Singh, S.V. and Chauhan, D.S. (2011) Electronically Tunable Low Voltage Mixed-Mode Universal Biquad Filter. IET Circuits, Devices and Systems, 5, 149-158. http://dx.doi.org/10.1049/iet-cds.2010.0061

[18] Maheshwari, S. and Khan, I.A. (2004) Current Controlled Current Differencing Buffered Amplifier: Implementation and Applications. Active and Passive Electronic Components, 4, 219-227. http://dx.doi.org/10.1080/08827510310001648924

[19] Satansup, J. and Tangsrirat, W. (2013) Single VDTA-Based Current-Mode Electronically Tunable Multifunction Filter. Proceedings of the 4th International Science, Social Science, Engineering and Energy, 1-8.

[20] Prasad, D., Bhaskar, D.R. and Srivastava, M. (2013.) Universal Current-Mode Biquad Filter Using a VDTA. Circuits and Systems, 4, 29-33. http://dx.doi.org/10.4236/cs.2013.41006

[21] Satansup, J., Pukkalanun, T. and Tangsrirat, W. (2013) Electronically Tunable Current-Mode Universal Filter Using VDTAs and Grounded Capacitors. Proceedings of the International Multi Conference of Engineers and Computer Scientists.

[22] Mekhum, W. and Jaikla, W. (2013) Three Input Single Output Voltage-Mode Multifunction Filter with Independent Control of Pole Frequency and Quality Factor. Theoretical and Applied Electrical Engineering, 11, 494-500.

[23] Prasad, D., Bhaskar, D.R. and Srivastava, M. (2013) Universal Voltage-Mode Biquad Filter Using Differencing Transconductance Amplifier. International Journal of Pure and Applied Physics, 51, 864-868.

[24] Yeşil, A., Kaçar, F. and Kuntman, H. (2011) New Simple CMOS Realization of Voltage Differencing Transconductance Amplifier and Its RF Filter Application. Radioengineering, 20, 632-637.

[25] Satansup, J., Pukkalanun, T. and Tangsrirat, W. (2013) Electronically Tunable Single-Input Five-Output Voltage-Mode Universal Filter Using VDTAs and Grounded Passive Elements. International Journal of Circuits System and Signal Processing, 32, 945-957.

[26] Chumwangwapee, K., Jaikla, W., Sunthonkanokpong, W., Jaikhang, W., Maneewan, S. and Sreewirote, B. (2014) High Input Impedance Mixed-Mode Biquad Filter with Orthogonal Tune of Natural Frequency and Quality Factor. 4th Joint International Conference on JICTEE, Chiang Rai, 5-8 March 2014, 1-4.

[27] Stamness, R.L. (2010) Improvement of a Propagation Delay Model for CMOS Digital Logic Circuits. Master’s Thesis and Graduate Research, San Jose State University, San Jose. 